\title{
Echocardiography at Bedside For Predicting Mortality of COVID-19 Patients Beyond Clinical Data - Data From the PROVAR-COVID Study
}

\section{Sander Luiz Gomes Pimentel}

Hospital das Clínicas da Universidade Federal de Minas Gerais: Hospital das Clinicas da Universidade Federal de Minas Gerais

Bruno Ramos Nascimento ( $\square$ ramosnas@gmail.com )

Hospital das Clínicas da Universidade Federal de Minas Gerais https://orcid.org/0000-0002-5586-774X

\section{Juliane Franco}

Hospital das Clínicas da Universidade Federal de Minas Gerais: Hospital das Clinicas da Universidade

Federal de Minas Gerais

\section{Kaciane Krauss Bruno Oliveira}

Hospital das Clínicas da Universidade Federal de Minas Gerais: Hospital das Clinicas da Universidade

Federal de Minas Gerais

\section{Clara Leal Fraga}

Universidade Federal de Minas Gerais Faculdade de Medicina

Frederico Vargas Botinha Macedo

Universidade Federal de Minas Gerais Faculdade de Medicina

\section{Leonardo Arruda Moraes Raso}

Hospital das Clínicas da Universidade Federal de Minas Gerais: Hospital das Clinicas da Universidade Federal de Minas Gerais

\section{Renata Eliane de Ávila}

Hospital das Clínicas da Universidade Federal de Minas Gerais: Hospital das Clinicas da Universidade Federal de Minas Gerais

\section{Luiza Pereira Afonso Santos}

Universidade Federal de Minas Gerais Faculdade de Medicina

\section{Rodrigo Tavares Lanna Rocha}

Universidade Federal de Minas Gerais Faculdade de Medicina

\section{Renan Mello Oliveira}

Universidade Federal de Minas Gerais Faculdade de Medicina

\section{Márcia Melo Barbosa}

Hospital das Clínicas da Universidade Federal de Minas Gerais: Hospital das Clinicas da Universidade Federal de Minas Gerais

Craig Sable 
Children's National Medical Center: Children's National Hospital

\section{Antonio Luiz Pinho Ribeiro}

Hospital das Clínicas da Universidade Federal de Minas Gerais: Hospital das Clinicas da Universidade Federal de Minas Gerais

\section{Andrea Zawacki Beaton}

Cincinnati Children's Hospital Medical Center

\section{Maria Carmo Pereira Nunes}

Hospital das Clínicas da Universidade Federal de Minas Gerais: Hospital das Clinicas da Universidade Federal de Minas Gerais

\section{Research Article}

Keywords: COVID-19, echocardiography, prognosis, SARS-Cov-2, mortality

Posted Date: April 20th, 2021

DOl: https://doi.org/10.21203/rs.3.rs-435447/v1

License: (a) (1) This work is licensed under a Creative Commons Attribution 4.0 International License. Read Full License 


\section{Abstract \\ Purpose}

Cardiac involvement seems to impact prognosis of COVID-19, being more frequent in critically ill patients. We aimed to assess the prognostic value of right ventricular (RV) and left ventricular (LV) dysfunction, evaluated by bedside echocardiography (echo), in patients hospitalized with COVID-19.

\section{Methods}

Patients admitted in 2 reference hospitals in Brazil from Jul to Sept/2020 with confirmed COVID-19 and moderate/severe presentations underwent clinical and laboratory evaluation, and focused bedside echo (GE Vivid-IQ), at the earliest convenience, with remote interpretation. The association between demographics, clinical comorbidities and echo variables with all-cause hospital mortality was assessed, and factors significant at $p<0.10$ were put into multivariable models.

\section{Results}

Total 163 patients were enrolled, mean age was $64 \pm 16$ years, 59\% were men and 107 (66\%) were admitted to intensive care. Comorbidities were present in 144 (88\%) patients: hypertension $115(71 \%)$, diabetes $61(37 \%)$ and heart failure $22(14 \%)$. In-hospital mortality was $34 \%(\mathrm{~N}=56)$. In univariate analysis, echo variables significantly associated with death were: LV ejection fraction $(L V E F, O R=0.94)$, $R V$ fractional area change $(\mathrm{OR}=0.96)$, tricuspid annular plane systolic excursion (TAPSE, OR $=0.83$ ) and $R V$ dysfunction ( $O R=5.3)$. In multivariate analysis, after adjustment for clinical and demographic variables, independent predictors of mortality were age $(\mathrm{OR}=1.05,95 \% \mathrm{Cl} 1.01-1.10, p=0.023)$, LVEF (OR $=0.95,95 \% \mathrm{Cl} 0.91-1.00, \mathrm{p}=0.48)$ and TAPSE $(\mathrm{OR}=0.76,95 \% \mathrm{Cl} 0.63-0.91, \mathrm{p}=0.005)$. The final model had good discrimination, with $\mathrm{C}$-statistic $=0.78(95 \% \mathrm{Cl} 0.68-0.88)$.

\section{Conclusion}

Markers of RV and LV dysfunction assessed by bedside echo are independent predictors of mortality in hospitalized COVID-19 patients, after adjustment for clinical variables.

\section{Introduction:}

The severe acute respiratory syndrome coronavirus 2 (SARS-CoV-2) pandemic is having a striking impact on the Brazilian health, with almost 8 million cases and over 200,000 deaths through January 2021[1]. Besides the absolute number of cases and deaths directly attributable to the virus, the excess mortality a strategy to additionally evaluate the indirect impacts of the disease - was also remarkable in this period, including that associated with cardiovascular disease (CVD)[2]. 
There are ongoing investigations to define prognostic factors for patients with the new coronavirus disease (COVID-19), and risk factors (e.g. hypertension, diabetes, obesity), biomarkers (e.g. troponins, ddimer, inflammatory markers), in addition to heart failure and disease-related cardiac disease, are associated with poor outcomes $[3,4]$. Echocardiography (echo) emerged as a promising modality to improve outcome prediction in COVID-19, being simple, applicable at the bedside and with lower risk of contamination[5]. Echo-detected cardiac abnormalities are frequent in COVID-19: in a series with over 1,200 patients, 667 (55\%) had an abnormal echo, with left (LV) and right ventricular (RV) abnormalities reported in $39 \%$ and $33 \%$, respectively, and evidence of new myocardial infarction in 3\%[6]. In addition, markers of RV dysfunction associate with biomarkers of worse prognosis, as troponin and D-dimer, and were proposed as independent predictors of all-cause mortality [7, 8]. As such, echo screening for acute heart failure has been proposed for critically ill COVID-19 patients in international position statements[9].

The mechanisms behind such findings are still under investigation. It is known that COVID-19 affects the cardiovascular system by different pathways, including direct myocardial injury due to viral invasion, systemic inflammatory response and excessive cytokine release resulting in multiple-organ lesions, plaque rupture and thrombosis, and increased cardiometabolic demand[10-12]. Acute cardiac disease occurs in 8 to $20 \%$ of all patients[10-13].

Thus, prospective systematic studies on the impact of routine echo assessment are of utmost importance in the context of the COVID-19 pandemic. In this study we aimed to assess the prognostic value of several echocardiographic variables, evaluated by bedside echo, in patients with COVID-19 in Brazil who fulfilled criteria for hospitalization.

\section{Methods:}

The PROVAR + program has been conducted since 2017 in the state of Minas Gerais, southeast Brazil, under the auspices of the Universidade Federal de Minas Gerais and the Telehealth Network of Minas Gerais[14], in collaboration with the Children's National Health System, Washington - DC, USA and the Cincinnati Children's Hospital Medical Center, Cincinnati - OH, USA. The program utilizes portable and ultra-portable echo devices for imaging acquisition and remote interpretation by telemedicine in different settings. The study protocol conforms to the ethical guidelines of the 1975 Declaration of Helsinki, and ethics approval was obtained from the institutional review boards of Universidade Federal de Minas Gerais and Hospital Eduardo de Menezes. Data analytic methods and study materials will be made available to other researchers for purposes of reproducing the results or replicating the procedure, from the corresponding author upon reasonable request.

In this study, consecutive patients hospitalized in 2 reference hospitals (Hospital Eduardo de Menezes, Fundação Hospitalar do Estado de Minas Gerais, Belo Horizonte, MG, Brazil and Hospital das Clínicas da Universidade Federal de Minas Gerais, Belo Horizonte, MG, Brazil) with confirmed COVID-19 (positive realtime polymerase chain (RT-PCR) for SARS-CoV-2) and a spectrum of moderate and severe clinical presentation according to the Berlin definition[15] underwent a standardized and detailed clinical 
questionnaire and laboratory evaluation, including complete blood count, serology for COVID-19 (immunoenzymatic assay, IgM and IgG) and biochemistry (including liver and renal function tests and inflammatory markers), analysed in central labs. All patients underwent bedside echocardiography (Vivid IQ ${ }^{\circledR}$ and Vivid Q®, GE Healthcare, Milwaukee - WI, USA), with acquisition of standard parasternal and apical views by experienced cardiologists (SP, MCN, LMR) with local support of the study staff (nurses, physical therapist, medical students) for further remote consensus interpretation (MMB, MCN). Exams were performed at the earliest convenience following admission, considering clinical and technical issues to acquire interpretable images and medical priorities defined by the intensive care staff.

Echocardiographic images were uploaded to a dedicated reading system (EchoPAC $\circledast$, GE Healthcare) and reviewed by two expert cardiologists ( $\mathrm{MN}$ and $\mathrm{MMB}$ ), and discrepancies were consensually solved. The comprehensive echocardiographic protocol focused on LV and RV morphology and function, mitral, aortic and tricuspid valves, pulmonary artery pressure and morphology, segmental wall-motion abnormalities and pericardial effusion[14]. Echocardiographic measurements were performed offline according to the recommendations of the American Society of Echocardiography[16-18]. Global RV function was quantitatively assessed using fractional area change, peak systolic velocity at the tricuspid annulus using tissue Doppler imaging, and tricuspid annular plane systolic excursion (TAPSE) at the RV free-wall, obtained from 2-dimensionally-guided M-mode recordings. Systolic pulmonary artery pressure was measured with tricuspid regurgitant velocity. Diastolic function was assessed by pulsed-wave Doppler examination of mitral inflow, and by tissue Doppler imaging. Left ventricular ejection fraction (LVEF) was calculated per the modified Simpson method

or, in cases with poor images, by linear dimensions with 2D-guided measurements[16-18]. Objective and subjective observations were reported. Preliminary reports were promptly made available to the hospital for clinical care. The main COVID-19 unit (Eduardo de Menezes, a public hospital run by the State Board of Health) has very limited access to imaging and relied largely on echo screening for medical decisions, while Hospital das Clínicas is a quaternary university hospital with local computed tomography and magnetic resonance imaging.

All data were entered to a RedCap ${ }^{\circledR}$ database[19]. Statistical analysis was performed using SPSS ${ }^{\circledR}$ software version 23.0 for Mac OSX (SPSS Inc., Chicago, Illinois). Considering preliminary studies that suggest that approximately $20 \%$ of the population of patients infected with SARS-CoV-2 present some degree of RV involvement, a 95\% power and alpha error of 5\%, a minimum sample of 143 patients was calculated. Considering losses and data completeness issues, we included consecutive eligible patients admitted over 2 months (July and August, 2020), during the peak of the pandemic in Belo Horizonte. The primary outcome was all-cause in-hospital death. Data are presented for all patients with confirmed RTPCR for COVID-19: categorical variables, expressed as numbers and percentages were compared between groups (patients who recovered vs. those who died in the index hospitalization) using Fisher's exact test, whereas continuous data, expressed as mean \pm standard deviation (SD) or median and Q1 - Q3 (25\% $75 \%)$, were compared using Student's unpaired t-test or the Mann-Whitney $U$ test, as appropriate. The association between demographics (age, sex), clinical comorbidities and echo variables (Table 1) with 
the primary outcome was assessed in univariate logistic regression analysis, and factors significant at $p$ $<0.10$ were put into multivariable adjusted logistic models. Significant continuous variables in the final model were dichotomized, with optimal cut-offs defined from the Receiver Operating Characteristic (ROC) curves, and a new simplified model was adjusted. A two-tailed significance level of 0.05 was considered statistically significant.

\section{Results:}

At total 163 patients were consecutively enrolled, mean age was $64 \pm 16$ years, 96 (59\%) were men and 107 (66\%) had severe COVID-19 with admission to intensive care during the index hospitalization. The median time from symptom onset to admission was 7 [IQR 4.0-9.0] days. Table 1 depicts demographic, clinical and echocardiographic characteristics of the study sample. During hospitalization, 56 patients died, corresponding to an overall mortality rate of $34 \%$.

Clinical comorbidities were present in 144 (88\%) patients: hypertension in 115 (71\%), diabetes in 61 (37\%) and heart failure in $22(14 \%)$ and were similar among those who died and survived in the index hospitalization. Patients who recovered, however, were significantly younger (Table 1). The most frequent symptoms reported at admission were dyspnea $(N=132,81 \%)$, cough $(N=122,75 \%)$ and fever $(N=99$, $61 \%)$. Dyspnea was more frequently reported by patients who died and, conversely, taste loss was more prevalent among those with favorable outcome (Table 1).

The clinical course of patients who died was also less favorable, with a higher proportion of individuals requiring intubation and mechanical ventilation $(94.6 \%$ vs. $26.2 \%, p<0.001)$ and administration of vasopressors ( $91.1 \%$ vs. $19.6 \%, p<0.001$ (Table 1 ). In addition, this group had a higher burden of abnormalities in bedside echo, especially those associated with LV and RV function and dimensions. Moderate and severe mitral regurgitation, however, was more prevalent among survivors (Table 1). Pulmonary artery thrombosis was directly diagnosed in 1 case, with a mobile thrombus in the pulmonary artery (Fig. 1).

In univariate analysis, echo RV and LV function variables significantly associated with death: LVEF (Oddsratio (OR): $0.94,95 \% \mathrm{Cl} 0.92-0.98$ ), RV fractional area change (OR: $0.96,95 \% \mathrm{Cl} 0.93-1.00)$, TAPSE (OR: $0.83,95 \% \mathrm{Cl} 0.74-0.93$ ) and any degree of RV dysfunction (OR: $5.33,95 \% \mathrm{Cl} 2.33-12.21$ ), as well as age (HR:1.03, 95\% Cl 1.01-1.05), dyspnea at presentation (OR: 4.39, 95\% Cl 1.45-13.29) and taste loss (OR: $0.21,95 \% \mathrm{Cl} 0.05-0.97$ ) (Table 2). In multivariate analysis, after adjustment for clinical and demographic variables, independent predictors of mortality were age (OR: 1.05, 95\% $\mathrm{Cl} 1.01-1.10, p=0.023)$, LVEF (OR: 0.95, 95\% $\mathrm{Cl} 0.91-1.00, \mathrm{p}=0.048$ ) and TAPSE (OR: 0.76, 95\% Cl 0.63-0.91, $\mathrm{p}=0.005$ ) (Table 2). The final model had good overall discrimination, with C-statistic $=0.78(95 \% \mathrm{Cl} 0.68-0.88)(\mathrm{Fig} .2 \mathrm{~A})$. From the ROC curves, the optimal cut-offs and adjusted OR for the significant variables were: age $\geq 63$ years (OR: 5.53, 95\% $\mathrm{Cl} 1.52-20.17$ ), LVEF < 64\% (OR: 7.37, 95\% Cl 2.10-25.94) and TAPSE < $18.5 \mathrm{~mm}$ (OR: $9.43,95 \% \mathrm{Cl}$ 2.57-35.03), and the final simplified prediction model had C-statistic $=0.83(95 \% \mathrm{Cl} 0.75-0.91)$ (Fig. 2B). 


\section{Discussion:}

Our data, from a sample of inpatients with moderate and severe COVID-19 in Brazil, suggest that echo assessment at bedside may be useful as a prognostic tool, to improve early outcome prediction in the pandemic. Markers of RV dysfunction, combined with LVEF, predict mortality on top of known clinical predictors. Being practical and easily applicable, comprehensive bedside echo may improve the availability of early cardiac imaging, especially in resource-limited institutions.

Since the inception of the COVID-19 pandemic, and the high mortality observed in the elderly and in patients with significant clinical comorbidities[20,21], there have been efforts to define independent predictors of adverse and fatal outcomes, as well as to refine prediction models. Published data indicate that patients with preexisting CVD are at highest risk for complications[20], and resemble findings from previous epidemics, such as the SARS-CoV, MERS-CoV outbreaks, in which the association between preexistent CVD and disease-induced myocardial injury with worse outcomes was observed[22]. Thus, there seems to be a superposition of mechanisms directly linked to viral infection (e.g. cardiomyopathy induced by coronaviruses in animal models[23]), the stress of infection and a prothrombotic state, potentially inducing a quick decompensation in patients with heart failure or coronary artery disease[24]. In this sense, our data reinforces these assumptions, highlighting the impacts of ventricular impairment, especially for the RV.

Our sample consisted of moderate and severe cases requiring hospital admission and medical care (66\% intensive care), and such patients are a platform to look deeper into different aspects of the disease. Independently of whether COVID-19 exacerbates preexisting CVD or causes new structural/functional abnormalities[24], early detection of such conditions may be of value for patient management. Several symptoms of COVID-19 - such as chest pain and markedly dyspnea - overlap with those of acute $\operatorname{CVD}[25,26]$, and observational data suggest that electrocardiographic abnormalities and elevated biomarkers of cardiac injury are also prevalent, especially in severely ill individuals[20,24,27]. This, along with high cardiovascular risk profile of hospitalized individuals - as depicted by our sample - might trigger requests for echo for the great majority of patients. In institutions with limited imaging capabilities, such as one of our COVID-19 centers, this indication is even more frequent, in the absence of more advanced propaedeutic. Considering this, incorporation of echo in the management of COVID-19 patients who require hospital admission - regardless of underlying cardiac disease - into prognostic models is crucial.

On the other hand, despite the growing body of evidence about the utility of echo in COVID-19, the ideal imaging protocols need to be evaluated, given the shortage of personal protective equipment and the exposure of personnel. Considering the high-risk setting - especially in the intensive care environment we opted to apply a fast-track comprehensive protocol. Although simplified to optimize screening time, it allowed for a more accurate cardiac assessment compared to those proposed by recently published studies[8,28]. Thus, functional portable machines and even selected handheld devices can be utilized[29], improving availability and reducing costs. Beyond limiting the exposure time of the screener, 
non-experts (e.g. clinical personnel already in touch with COVID-19 patients) may be trained for imaging acquisition, and telemedicine may allow for remote interpretation. Similar equipment and strategy may be applied for point-of-care lung ultrasound (POCUS)[30]. Task-shifting, however, requires a learning curve, and the echo variables of interest must be carefully evaluated in this case.

Our key findings, suggesting RV dysfunction as a predictor of death, align with preliminary data from China and the UK, but these studies showed a relative LV sparing, in contrast with our observations[8, 31]. Regarding the choice of parameter for RV assessment, Li and Cols successfully demonstrated RV freewall strain as a more accurate predictor of unfavorable outcome, compared to other traditional variables[31]. This choice, however, conflicts with recommendations for simple and easily-applicable level-1 protocols in the pandemic[32, 33]. For this reason, we opted to systematically evaluate more practical and reproducible measures, as proposed by Moody and Argulian in the UK and the US, and supported by recent position statements[7, 8]. Thus, the RV variable independently associated with unfavorable outcome in this study was TAPSE, which has been previously correlated with acute-phase biomarkers as troponins and d-dimer[8]. The mechanisms behind this finding, however, are still a matter of debate, as RV dysfunction/enlargement may result from pulmonary vascular involvement and respiratory support.

In a European study in hospital settings, pulmonary hypertension, but not RV dysfunction, was associated with the composite outcome of death and/or indication for intensive care[34]. The study population, however, had much less severe presentations, with $4 \%$ requiring intubation and $<10 \%$ mortality, compared to over $66 \%$ intensive care admissions and $34 \%$ mortality in our sample[34]. Risk factors were also less prevalent. Thus, we hypothesize that infectious and inflammatory mechanisms presumably triggering RV impairment[8] were more intensive, and baseline cardiac disease more prevalent. In our study, the absence of elevated tricuspid velocity suggests mechanisms other than pulmonary hypertension to explain RV dysfunction. Indeed, RV function is determined by intrinsic RV contractility and ventricular preload/afterload. Therefore, its dysfunction in the setting of COVID-19 is likely related to several mechanisms, besides elevated pulmonary artery pressure.

In addition, pulmonary pressure is multifactorial - with marked variations in response to hypoxemiarelated vasoconstriction, increased PEEP and other ventilator parameters[35] - being more prone to bias in prediction models. Although pulmonary thrombosis resulting from hypercoagulation has been described in COVID-19[34], chest CT and lung scintigraphy were not routinely performed in our patients, and proximal thrombus was observed in only 1 case (Fig. 1).

Regarding the LV involvement observed in our data, it has been previously reported in other viral infections with intense systemic manifestations, such as Dengue[36], Zika[37], Chikugunya[38] and Yellow Fever[39], in their severe forms, often associated with impaired systolic function. As with the RV involvement, it remains unclear if this results from direct viral infection or replication leading to cytokine dysregulation[40] - mechanisms involved in severe COVID-19[4, 21] - or derives from hemodynamic instability and shock decompensating underlying disease. Pathological evidence of myocarditis has also 
been described[41]. As expected, a much higher proportion of patients with unfavourable outcome in our study (the subset with lower LVEF) required haemodynamic support with vasopressors (Table 1). For both LVEF and TAPSE, the conservative cut-offs in our final dichotomous prediction model were presumably associated with the hyperdynamic state of patients with moderate and severe COVID-19, resulting in tachycardia and high output.

In our sample, traditional cardiovascular risk factors for poor outcomes in epidemiological studies[4, 21] were not associated with mortality and were evenly distributed between survivors and those who died (Table 1). This may be due to our patient selection, consisting only of individuals requiring hospitalization with at least moderate symptoms, what may hinder the risk profile gradient between groups. For this reason, our model may not be generalizable to populations of mildly symptomatic patients. It is noticeably, however, that echo parameters remained as predictors even adjusted for clinical variables. Finally, more than investigating predictive parameters, our program, with a fast-track imaging routine, improved access to healthcare, as we prioritized screening for those who required immediate echo information - as indicated by the medical staff - what potentially facilitated lifesaving interventions in the short run. Studies looking for outcomes, however, are warranted. With refinement of the model, this experience from a Latin American country can be replicated to other under sourced regions.

\section{Limitations:}

Our study has several limitations. At first, due to logistics at each hospital and the severity of cases, echos were performed at the earliest convenience following admission, and not at a standardized time point after severe symptoms presented. For this reason, results may reflect different stages of systemic inflammation and cardiac involvement. Second, no serial exams were done, precluding conclusions about the longitudinal progression/regression of cardiac abnormalities in the course of COVID-19. Third, we opted to include patients with different degrees of oxygen supply and hemodynamic support, including those under mechanical ventilation and/or vasopressors at enrolment. Thus, hemodynamic responses to variable pulmonary pressures, including high positive end-expiratory pressure, and to different patterns of systemic and pulmonary vascular resistances, may impact echo variables. Finally, as severely ill patients were included, sometimes transferred from pre-hospital emergency units, clinical data was prone to imprecision, being frequently informed by relatives or companions. Data collection was pragmatic and, as a result, variables as Chagas disease - an endemic infectious condition in Brazil that may affect right heart chambers - were not systematically collected. However, our data - a pioneer analysis from a Latin American sample - reflect the real-life approach to patients with moderate to severe COVID-19, and the clinical variability may reflect the heterogeneity observed in the management of such cases. Our model suggests that, even with the aforementioned heterogeneity, bedside focused echo is a useful tool for risk stratification, with predictive value on top of clinical presentation.

\section{Conclusion:}


Markers of RV and LV dysfunction evaluated by focused bedside echo are independent predictors of mortality in hospitalized COVID-19 patients, after adjustment for clinical variables. Echocardiographic assessment of ventricular function can be potentially helpful for clinical risk stratification early following admission of patients with COVID-19, being a simple and widely available tool in the pandemic, especially in resource-limited settings.

\section{Declarations}

\section{Funding:}

The PROVAR+ study is funded Edwards Lifesciences Foundation ${ }^{\circledR}$, US, with complementary funding by FAPEMIG (Fundação de Amparo à Pesquisa do Estado de Minas Gerais). Echocardiography equipment is provided by General Electric Healthcare ${ }^{\circledR}$. The Telehealth Network of Minas Gerais was funded by the State Government of Minas Gerais, by its Health Department (Secretaria de Estado da Saúde de Minas Gerais) and FAPEMIG (Fundação de Amparo à Pesquisa de Minas Gerais), and by the Brazilian Government, including the Health Ministry and the Science and Technology Ministry and its research and innovation agencies, CNPq (Conselho Nacional de Desenvolvimento Científico e Tecnológico) e FINEP (Financiadora de Estudos e Projetos). Dr. Ribeiro was supported in part by CNPq (310679/2016-8 and 465518/2014-1), by FAPEMIG (PPM-00428-17 and RED-00081-16) and CAPES (88887.507149/2020-00). Dr. Nascimento was supported in part by CNPq (Bolsa de produtividade em pesquisa, 312382/2019-7). Medical students received scholarships from the National Institute of Science and Technology for Health Technology Assessment (IATS, project: 465518/2014-1).

\section{Conflicts of interest:}

The authors report no relationships that could be construed as a conflict of interest.

\section{Availability of data and material:}

Data analytic methods and study materials will be made available to other researchers for purposes of reproducing the results or replicating the procedure, from the corresponding author upon reasonable request.

\section{Authors contributions:}

Conception and design of the research: Nascimento, BR, Sable, C, Beaton, AZ, Nunes, MCP, Ribeiro AL; Acquisition of data: Pimentel, SLG, Oliveira, KKB, Franco, J, Barbosa, MM, Fraga, CL, Macedo, FVB, Raso, LAM, Ávila, RE, Santos, LPA, Rocha, RTL, Oliveira, RM; Analysis and interpretation of data: Pimentel, SLG , Nascimento, BR, Nunes, MCP, Sable, C, Beaton, AZ; Statistical analysis: Nascimento, BR, Nunes, MCP; Obtaining financing: Beaton, AZ, Sable, C, Nascimento, BR; Writing of the manuscript: Nascimento, BR, Pimentel, SLG; Critical revision of the manuscript for intellectual content: All authors; Authors responsible for the overall content as guarantors: Nascimento, BR, Beaton, AZ, Ribeiro, AL, Sable, C. 


\section{Ethics approval:}

The study protocol conforms to the ethical guidelines of the 1975 Declaration of Helsinki, and ethics approval was obtained from the institutional review boards of Universidade Federal de Minas Gerais and Hospital Eduardo de Menezes.

\section{Consent to participate:}

All patients included signed the informed consent prior to enrollment.

\section{Consent to publish:}

I would like to assure you that all authors participated actively in this study. All of them have seen and approved the submitted manuscript, which reports unpublished work not under consideration elsewhere.

\section{References}

[1] Worldometer. Worldometer - Coronavirus. COVID-19 Coronavirus Pandemic2020.

[2] Brant LCC, Nascimento BR, Teixeira RA, Lopes M, Malta DC, Oliveira GMM, et al. Excess of cardiovascular deaths during the COVID-19 pandemic in Brazilian capital cities. Heart. 2020;106:1898905.

[3] Wendel Garcia PD, Fumeaux T, Guerci P, Heuberger DM, Montomoli J, Roche-Campo F, et al. Prognostic factors associated with mortality risk and disease progression in 639 critically ill patients with COVID-19 in Europe: Initial report of the international RISC-19-ICU prospective observational cohort. EClinicalMedicine. 2020;25:100449.

[4] Izcovich A, Ragusa MA, Tortosa F, Lavena Marzio MA, Agnoletti C, Bengolea A, et al. Prognostic factors for severity and mortality in patients infected with COVID-19: A systematic review. PloS one. 2020;15:e0241955.

[5] Capotosto L, Nguyen BL, Ciardi MR, Mastroianni C, Vitarelli A. Heart, COVID-19, and echocardiography. Echocardiography. 2020;37:1454-64.

[6] Dweck MR, Bularga A, Hahn RT, Bing R, Lee KK, Chapman AR, et al. Global evaluation of echocardiography in patients with COVID-19. European heart journal cardiovascular Imaging. 2020;21:949-58.

[7] Argulian E, Sud K, Vogel B, Bohra C, Garg VP, Talebi S, et al. Right Ventricular Dilation in Hospitalized Patients With COVID-19 Infection. JACC Cardiovasc Imaging. 2020;13:2459-61.

[8] Moody WE, Mahmoud-Elsayed HM, Senior J, Gul U, Khan-Kheil AM, Horne S, et al. Impact of Right Ventricular Dysfunction on Mortality in Patients Hospitalized with COVID-19 according to Race. CJC 
Open. 2020.

[9] Lal S, Hayward CS, De Pasquale C, Kaye D, Javorsky G, Bergin P, et al. COVID-19 and Acute Heart Failure: Screening the Critically III - A Position Statement of the Cardiac Society of Australia and New Zealand (CSANZ). Heart, lung \& circulation. 2020;29:e94-e8.

[10] Bansal M. Cardiovascular disease and COVID-19. Diabetes Metab Syndr. 2020;14:247-50.

[11] Li B, Yang J, Zhao F, Zhi L, Wang X, Liu L, et al. Prevalence and impact of cardiovascular metabolic diseases on COVID-19 in China. Clinical research in cardiology : official journal of the German Cardiac Society. 2020;109:531-8.

[12] Lippi G, Plebani M. Laboratory abnormalities in patients with COVID-2019 infection. Clin Chem Lab Med. 2020.

[13] Szekely Y, Lichter Y, Taieb P, Banai A, Hochstadt A, Merdler I, et al. Spectrum of Cardiac Manifestations in COVID-19: A Systematic Echocardiographic Study. Circulation. 2020;142:342-53.

[14] Nascimento BR, Beaton AZ, Nunes MCP, Tompsett AR, Oliveira KKB, Diamantino AC, et al. Integration of echocardiographic screening by non-physicians with remote reading in primary care. Heart. 2018.

[15] Force ADT, Ranieri VM, Rubenfeld GD, Thompson BT, Ferguson ND, Caldwell E, et al. Acute respiratory distress syndrome: the Berlin Definition. JAMA. 2012;307:2526-33.

[16] American Society of Echocardiography (ASE) Guidelines. In: Echocardiography ASo, editor. ASE Guidelines. Durham - NC: ASE; 2018.

[17] Zoghbi WA, Adams D, Bonow RO, Enriquez-Sarano M, Foster E, Grayburn PA, et al. Recommendations for Noninvasive Evaluation of Native Valvular Regurgitation: A Report from the American Society of Echocardiography Developed in Collaboration with the Society for Cardiovascular Magnetic Resonance. Journal of the American Society of Echocardiography : official publication of the American Society of Echocardiography. 2017;30:303-71.

[18] Mitchell C, Rahko PS, Blauwet LA, Canaday B, Finstuen JA, Foster MC, et al. Guidelines for Performing a Comprehensive Transthoracic Echocardiographic Examination in Adults: Recommendations from the American Society of Echocardiography. Journal of the American Society of Echocardiography : official publication of the American Society of Echocardiography. 2019;32:1-64.

[19] Harris PA, Taylor R, Thielke R, Payne J, Gonzalez N, Conde JG. Research electronic data capture (REDCap)-a metadata-driven methodology and workflow process for providing translational research informatics support. J Biomed Inform. 2009;42:377-81.

[20] Shi S, Qin M, Shen B, Cai Y, Liu T, Yang F, et al. Association of Cardiac Injury With Mortality in Hospitalized Patients With COVID-19 in Wuhan, China. JAMA Cardiol. 2020;5:802-10. 
[21] Zhou F, Yu T, Du R, Fan G, Liu Y, Liu Z, et al. Clinical course and risk factors for mortality of adult inpatients with COVID-19 in Wuhan, China: a retrospective cohort study. Lancet. 2020;395:1054-62.

[22] Sellers SA, Hagan RS, Hayden FG, Fischer WA, 2nd. The hidden burden of influenza: A review of the extra-pulmonary complications of influenza infection. Influenza Other Respir Viruses. 2017;11:372-93.

[23] Alexander LK, Small JD, Edwards S, Baric RS. An experimental model for dilated cardiomyopathy after rabbit coronavirus infection. J Infect Dis. 1992;166:978-85.

[24] Kochi AN, Tagliari AP, Forleo GB, Fassini GM, Tondo C. Cardiac and arrhythmic complications in patients with COVID-19. J Cardiovasc Electrophysiol. 2020;31:1003-8.

[25] Tahir F, Bin Arif T, Ahmed J, Malik F, Khalid M. Cardiac Manifestations of Coronavirus Disease 2019 (COVID-19): A Comprehensive Review. Cureus. 2020;12:e8021.

[26] Basu-Ray I, Almaddah N, Adeboye A, Soos MP. Cardiac Manifestations Of Coronavirus. StatPearls. Treasure Island (FL)2020.

[27] Long B, Brady WJ, Bridwell RE, Ramzy M, Montrief T, Singh M, et al. Electrocardiographic manifestations of COVID-19. Am J Emerg Med. 2020;41:96-103.

[28] Rodriguez-Santamarta M, Minguito-Carazo C, Echarte-Morales JC, Del Castillo-Garcia S, Valdivia-Ruiz J, Fernandez-Vazquez F. Echocardiographic findings in critical patients with COVID-19. Rev Esp Cardiol (Engl Ed). 2020;73:861-3.

[29] Jenkins S, Garg P. Prime time for handheld echocardiography in COVID-19 pandemic. Clin Med (Lond). 2020;20:e132.

[30] Picard MH, Weiner RB. Echocardiography in the Time of COVID-19. Journal of the American Society of Echocardiography : official publication of the American Society of Echocardiography. 2020;33:674-5.

[31] Li Y, Li H, Zhu S, Xie Y, Wang B, He L, et al. Prognostic Value of Right Ventricular Longitudinal Strain in Patients With COVID-19. JACC Cardiovasc Imaging. 2020;13:2287-99.

[32] Jozsa C, Ussen B, Monteiro R, Bingcang R, Lloyd G, Bhattacharyya S. Impact of Focused Echocardiography on Scan Time and Diagnostic Quality in Patients with COVID-19. Journal of the American Society of Echocardiography : official publication of the American Society of Echocardiography. 2020;33:1415-6.

[33] Ward RP, Lee L, Ward TJ, Lang RM. Utilization and Appropriateness of Transthoracic Echocardiography in Response to the COVID-19 Pandemic. Journal of the American Society of Echocardiography : official publication of the American Society of Echocardiography. 2020;33:690-1. 
[34] Pagnesi M, Baldetti L, Beneduce A, Calvo F, Gramegna M, Pazzanese V, et al. Pulmonary hypertension and right ventricular involvement in hospitalised patients with COVID-19. Heart. 2020;106:1324-31.

[35] Garcia-Cruz E, Manzur-Sandoval D, Rascon-Sabido R, Gopar-Nieto R, Barajas-Campos RL, JordanRios A, et al. Critical care ultrasonography during COVID-19 pandemic: The ORACLE protocol. Echocardiography. 2020;37:1353-61.

[36] Nagaratnam N, Siripala K, de Silva N. Arbovirus (dengue type) as a cause of acute myocarditis and pericarditis. British heart journal. 1973;35:204-6.

[37] Minhas AM, Nayab A, lyer S, Narmeen M, Fatima K, Khan MS, et al. Association of Zika Virus with Myocarditis, Heart Failure, and Arrhythmias: A Literature Review. Cureus. 2017;9:e1399.

[38] Alvarez MF, Bolivar-Mejia A, Rodriguez-Morales AJ, Ramirez-Vallejo E. Cardiovascular involvement and manifestations of systemic Chikungunya virus infection: A systematic review. F1000Res. 2017;6:390.

[39] Paixao GMM, Nunes MCP, Beato B, Sable C, Beaton AZ, Oliveira KKB, et al. Cardiac Involvement by Yellow Fever(from the PROVAR+ Study). Am J Cardiol. 2019;123:833-8.

[40] Silva ML, Espirito-Santo LR, Martins MA, Silveira-Lemos D, Peruhype-Magalhaes V, Caminha RC, et al. Clinical and immunological insights on severe, adverse neurotropic and viscerotropic disease following 17D yellow fever vaccination. Clinical and vaccine immunology : CVI. 2010;17:118-26.

[41] Siripanthong B, Nazarian S, Muser D, Deo R, Santangeli P, Khanji MY, et al. Recognizing COVID-19related myocarditis: The possible pathophysiology and proposed guideline for diagnosis and management. Heart Rhythm. 2020;17:1463-71.

\section{Tables}

Table 1: Demographic, clinical and echocardiographic characteristics of patients who survived and died during the index hospitalization. 
Variable:

Variable:

Demographics and risk factors:

Age (median, [IQR])

Sex (male, $N(\%))$ :

Any clinical comorbidities ( $N, \%)$

Hypertension ( $N$, \%):

Diabetes (N, \%):

Heart failure ( $N, \%)$ :

Obesity ( $N, \%)$ :

Asthma (N, \%):

Smoking ( $N$, \%):

COVID-19 symptoms at admission:

Symptom onset to admission (days, median [IQR])

Fever at admission $\left(>37.8^{\circ} \mathrm{C}\right)(\mathrm{N}, \%)$ :

Cough ( $N$, \%):

Dyspnea ( $\mathrm{N}, \%)$ :

Diarrhea ( $N$, \%):

Headache $(\mathrm{N}, \%)$

Abdominal pain $(\mathrm{N}, \%)$ :

Anosmia (N, \%):

Taste loss ( $\mathrm{N}, \%)$ :

Respiratory support and vasopressors:

Noninvasive ventilation $(\mathrm{N}, \%)$

Mechanical ventilation ( $N$, \%)

Days in mechanical ventilation (median, [IQR])

Tracheostomy ( $\mathrm{N}, \%)$

Use of vasopressors ( $\mathrm{N}, \%)$

Echo variables:
Survivors

$(\mathrm{N}=107)$
Hospital death

$(\mathrm{N}=56)$
Pvalue:
$62.0[52.2-$
$71.5)$

$63(58.9)$

$93(86.9)$

$75(70.1)$

36 (33.6)

$15(14.1)$

$13(12.1)$

$5(4.7)$

$26(24.3)$

$7.0[4.0-9.0]$

$65(60.7)$

$80(74.8)$

$80(74.7)$

19 (17.8)

$14(13.1)$

5 (4.7)

17 (15.9)

18 (16.8)

14 (13.1)

28 (26.2)

13.0 [6.0 - 23.0]

$10(9.3)$

21 (19.6)

$68.2[61.8-78.8]$

0.01 *

33 (58.9)

0.40

51 (91.1)

0.61

40 (71.4)

1.00

25 (44.6)

0.22

7 (12.5)

0.81

$5(8.9)$

0.79

4 (7.1)

0.72

$13(23.2)$

1.00

$7.0[5.0-11.0]$

0.43

$34(60.7)$

0.74

$42(75.0)$

1.00

52 (92.9)

0.006 *

4 (7.1)

0.10

$3(5.4)$

0.27

$3(5.4)$

1.00

3 (5.4)

0.12

2 (3.6)

$0.04^{*}$ 


\begin{tabular}{|c|c|c|c|}
\hline Admission to echo (days, median [IQR]) & $6.0[3.0-9.0]$ & $7.0[3-12]$ & 0.20 \\
\hline LVEF (\%) & $\begin{array}{l}65.0[61.0- \\
70.0]\end{array}$ & $61.0[46.0-66.0]$ & $<0.001^{*}$ \\
\hline LV diastolic diameter & $\begin{array}{l}45.0[43.0- \\
49.0]\end{array}$ & $45.0[40.0-49.0]$ & 0.21 \\
\hline LV systolic diameter & $\begin{array}{l}29.0[26.0- \\
32.0]\end{array}$ & $32[28.0-36.0]$ & $0.008 *$ \\
\hline RV dysfunction (any) (N, \%) & $11(10.3)$ & $21(37.5)$ & $<0.001^{*}$ \\
\hline LA diameter $(\mathrm{mm})(\operatorname{mean} \pm \mathrm{SD})$ & $37.9 \pm 4.7$ & $36.3 \pm 6.1$ & 0.084 \\
\hline RV fractional area change (\%) & $39.8 \pm 10.0$ & $35.6 \pm 11.4$ & $0.046 *$ \\
\hline TAPSE (mm) & $\begin{array}{l}20.0[18.0- \\
23.0]\end{array}$ & $16.0[14.0-19.0]$ & $<0.001^{*}$ \\
\hline RV basal dimension (mm) (median [IQR]) & $\begin{array}{l}34.0[31.0- \\
38.0]\end{array}$ & $38.0[36.0-43.0]$ & $<0.001^{*}$ \\
\hline Septal E/E' & $9.3[8.0-11.8]$ & $10.3[9.2-13.0]$ & 0.08 \\
\hline Tricuspid velocity (m/s) (mean $\pm S D)$ & $2.8 \pm 0.4$ & $3.0 \pm 0.4$ & 0.19 \\
\hline Mitral regurgitation (moderate/severe) $(\mathrm{N}, \%)$ : & $12(11.2)$ & $1(1.8)$ & $0.007 *$ \\
\hline LV wall-motion abnormalities ( $\mathrm{N}, \%)$ : & $7(6.5)$ & $6(10.7)$ & 0.37 \\
\hline Pericardial effusion ( $N, \%)$ : & $15(14.0)$ & $10(17.8)$ & 0.49 \\
\hline
\end{tabular}

Abbreviations: LA: left atrium; LV: left ventricle; LVEF: left ventricular ejection fraction; OR: odds-ratio; RV: right ventricle; TAPSE: tricuspid annular plane systolic excursion.

Table 2: Univariable and multivariable analysis of demographic, clinical and echocardiographic variables associated with in-hospital mortality. 


\begin{tabular}{|c|c|c|c|}
\hline \multicolumn{4}{|l|}{ Univariable analysis: } \\
\hline Variable: & OR: & 95\% Cl: & P-value: \\
\hline \multicolumn{4}{|l|}{ Demographics and risk factors: } \\
\hline Age (each 1 year): & 1.03 & $1.01-1.05$ & $0.013^{*}$ \\
\hline Sex (male): & 1.41 & $0.72-2.75$ & 0.31 \\
\hline Hypertension: & 0.98 & $0.41-2.28$ & 0.93 \\
\hline Diabetes: & 1.58 & $0.79-3.17$ & 0.19 \\
\hline Heart failure: & 0.85 & $0.32-2.24$ & 0.74 \\
\hline Obesity: & 0.73 & $0.24-2.24$ & 0.59 \\
\hline Asthma: & 1.50 & $0.38-5.85$ & 0.56 \\
\hline Smoking: & 0.94 & $0.44-2.02$ & 0.88 \\
\hline \multicolumn{4}{|c|}{ COVID-19 symptoms at admission: } \\
\hline Fever: & 0.89 & $0.46-1.72$ & 0.73 \\
\hline Cough: & 1.09 & $0.51-2.33$ & 0.82 \\
\hline Dyspnea: & 4.39 & $1.45-13.29$ & $0.009 *$ \\
\hline Diarrhea: & 0.80 & $0.11-1.13$ & 0.08 \\
\hline Abdominal pain: & 1.16 & $0.27-5.07$ & 0.84 \\
\hline Anosmia: & 0.35 & $0.10-1.28$ & 0.11 \\
\hline Taste loss: & 0.21 & $0.05-0.97$ & $0.045^{*}$ \\
\hline \multicolumn{4}{|l|}{ Echo variables: } \\
\hline LVEF (\%) & 0.94 & $0.92-0.98$ & $<0.001 *$ \\
\hline RV dysfunction (any) & 5.33 & $2.33-12.21$ & $<0.001 *$ \\
\hline LA diameter (mm) & 0.94 & $0.88-1.00$ & 0.06 \\
\hline RV fractional area change (\%) & 0.96 & $0.93-1.00$ & $0.049 *$ \\
\hline TAPSE (mm) & 0.83 & $0.74-0.93$ & $0.001 *$ \\
\hline RV basal dimension (mm) & 1.10 & $1.03-1.18$ & $0.004^{*}$ \\
\hline Septal E/E' & 1.07 & $0.97-1.17$ & 0.20 \\
\hline Tricuspid velocity (m/s) & 2.60 & $0.68-9.92$ & 0.16 \\
\hline Multivariable analysis $t:$ & & & \\
\hline
\end{tabular}




\begin{tabular}{|llll|} 
Age (each 1 year): & 1.05 & $1.01-1.10$ & $\mathbf{0 . 0 2 3}$ \\
\hline LVEF (\%): & 0.96 & $0.91-1.00$ & $\mathbf{0 . 0 4 8 *}$ \\
\hline TAPSE $(\mathbf{m m})$ & 0.76 & $0.63-0.91$ & $\mathbf{0 . 0 0 5 *}$ \\
\hline
\end{tabular}

Abbreviations: LA: left atrium; LVEF: left ventricular ejection fraction; OR: odds-ratio; RV: right ventricle; TAPSE: tricuspid annular plane systolic excursion. * $p<0.05$. † Multivariable analysis adjusted for sex, hypertension, diabetes, heart failure, asthma, obesity, fever, cough.

\section{Figures}
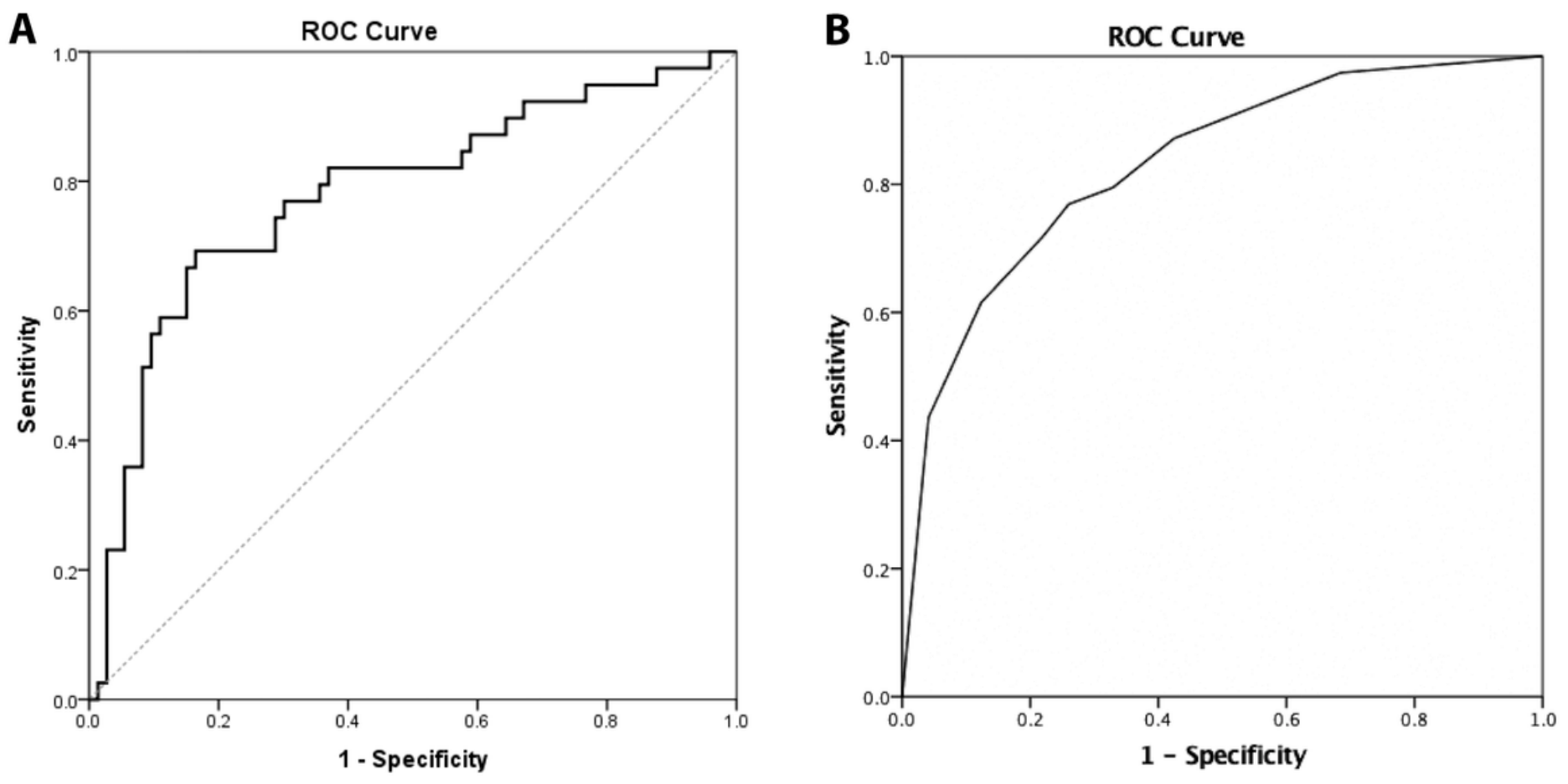

\section{Figure 1}

Echocardiographic images of a young (42 years-old) male patient who evolved with severe COVID-19, showing RV enlargement (A) with a D-shaped LV (B), a proximal mobile thrombus in the main pulmonary artery (C) and a dilated IVC (D). This patient died during the index hospitalization. IVC: inferior vena cava; LA: left atrium; LV: left ventricle; PA: pulmonary artery; RA: right atrium; RV: right ventricle. 


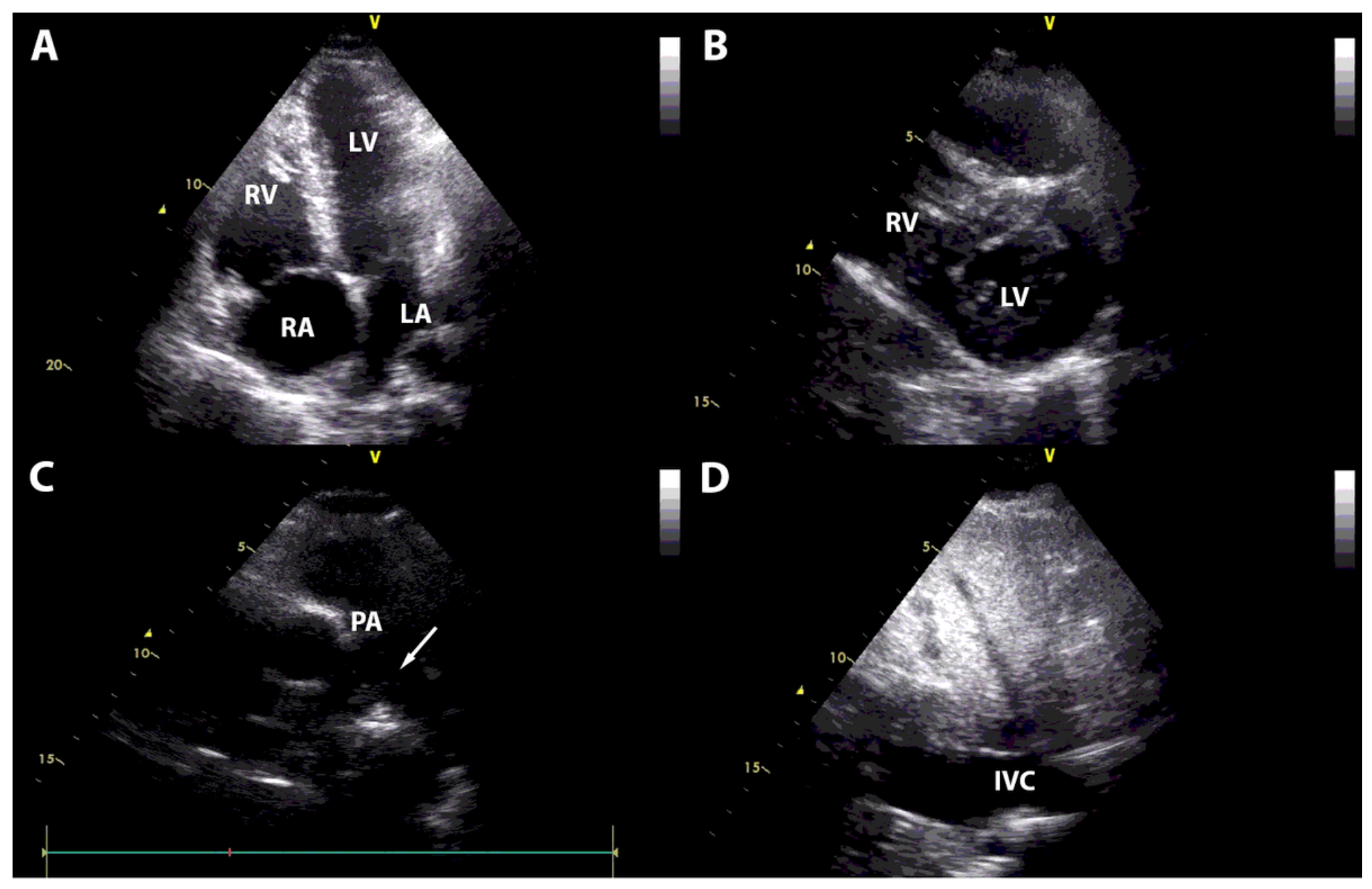

Figure 2

A. Receiver Operating Characteristic (ROC) curve of the model including the significant variables in the multivariate logistic model to predict in-hospital mortality for patients with COVID-19; C-statistic $=0.78$ ( $95 \% \mathrm{Cl} 0.68-0.88)$. B: ROC curve of the simplified model with dichotomized predictors of in-hospital mortality (age $\geq 63$ years, LVEF $<64 \%$ and TAPSE $<18.5 \mathrm{~mm}$ ); C-statistic $=0.83(95 \% \mathrm{Cl} 0.75-0.91)$. 